\title{
Rayleigh-wave attenuation by a semi-infinite two-dimensional elastic-band-gap crystal
}

\author{
F. Meseguer* and M. Holgado \\ Unidad Asociada Consejo Superior de Investigaciones Científicas, Departamento de Física Aplicada, \\ Universidad Politécnica de Valencia, Camino de Vera, E-46022 Valencia, Spain \\ and Instituto de Ciencia de Materiales, Consejo Superior de Investigaciones Científicas, \\ Campus de Cantoblanco, E-28049 Madrid, Spain \\ D. Caballero \\ Departamento de Física Teórica de la Materia Condensada, Facultad de Ciencias (C-V), Universidad Autonoma de Madrid, \\ E-28049 Madrid, Spain \\ N. Benaches \\ Unidad Asociada Consejo Superior de Investigaciones Científicas, Departamento de Física Aplicada, \\ Universidad Politécnica de Valencia, Camino de Vera, E-46022 Valencia, Spain \\ J. Sánchez-Dehesa ${ }^{\dagger}$ \\ Departamento de Física Teórica de la Materia Condensada, Facultad de Ciencias (C-V), Universidad Autonoma de Madrid, \\ E-28049 Madrid, Spain \\ C. López \\ Instituto de Ciencia de Materiales, Consejo Superior de Investigaciones Científicas, Campus de Cantoblanco, E-28049 Madrid, Spain \\ J. Llinares \\ Departamento de Física Aplicada, \\ Universidad Politécnica de Valencia, Camino de Vera, E-46022 Valencia, Spain \\ (Received 4 February 1999)
}

\begin{abstract}
In this paper, we report experiments on the scattering of surface-elastic waves by a periodic array of cylindrical holes. The experiments were performed in a marble quarry by drilling cylindrical holes in two different configurations: honeycomb and triangular lattices. The attenuation spectra of the surface waves show the existence of absolute band gaps for elastic waves in these semi-infinite two-dimensional crystals. Results are compared with theoretical calculations based on a scalar-wave approach. The scaling property of the underlying theory has led us to explore the possible application of the results obtained to the attenuation of surface waves in seismic movements. [S0163-1829(99)07419-6]
\end{abstract}

The study of photonic-band-gap (PBG) crystals is a field in which intensive research is being conducted due to their potential usefulness in nanotechnology. ${ }^{1}$ The underlying theory ${ }^{2}$ can be extended to other kinds of classical waves, like sound or elastic waves, in order to build up their corresponding crystals. These "classical crystals" can be called wave crystals (WC), with the appropriate gaps being photonic, sonic (SBG), or elastic (EBG) crystals. A common characteristic of these systems is the presence of two materials with different properties: one acts as the host for the wave propagation, and the other provides the scattering centers. The parameters that allow the appearance of full band gaps are: (i) the ratio between the magnitudes defining the scatterers and host (i.e., dielectric constants in PBG, densities and sound velocities in SBG, and elastic constants in EBG), (ii) the fraction of volume occupied by the scattering material, and (iii) the geometrical order of the scatterers inside the host.

The study of SBG and EBG materials have their own interest because of their possible application in sound filters and in vibrationless environments. Most of the work concerning these materials consists of theoretical calculations, ${ }^{3-7}$ and very few are experimental..$^{8-11}$

One property of paramount importance in WC theory is that the equations governing the wave propagation are scaleable. From the experimental side, this property allows work with manageable samples in a laboratory environment. Nevertheless, the realization of the crystal with the desirable dimensions is sometimes a difficult task; a paradigmatic example is the still unsolved construction of a threedimensional (3D) PBG with the band gap appropriate for visible light as predicted by Yablonovich. ${ }^{12}$

Elastic waves propagating at the interface of two different media is a topic of great importance since surface waves can affect our everyday life. In geophysics, Rayleigh and Love surface waves are excited when bulk waves, originating in seismic movements, reach the earth surface $;^{13}$ the Rayleigh waves are highly destructive. To the best of our knowledge, the most widely studied mechanism for surface-wave attenu- 

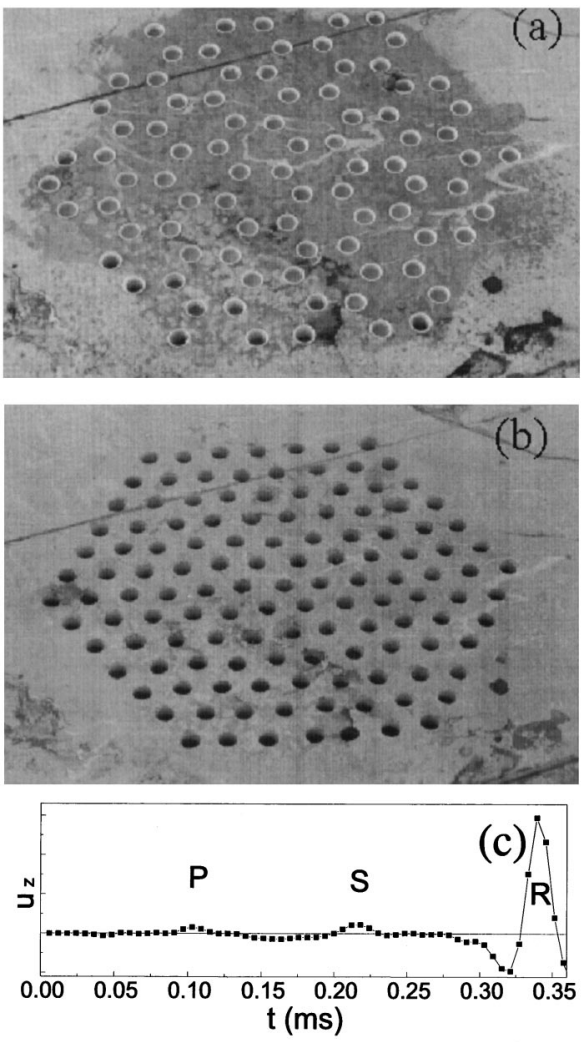

FIG. 1. (a) Photograph of the honeycomb structure of 6-cm diameter cylindrical holes, 14-cm first-neighbor distance, on the surface of the marble quarry. (b) Same for the triangular structure. (c) A typical seismogram produced by the impact of the bearing ball. The arrival of the primary (P) and secondary (S) waves can be observed before the Rayleigh wave $(\mathrm{R})$ reaches the detector.

ation is surface roughness. ${ }^{14}$ However, neither theory nor experiments, concerning the possible attenuation of surface waves by an EBG, have been reported.

In this paper, we present the study of the attenuation of surface-elastic waves by a collection of cylindrical holes periodically distributed on the surface of a marble quarry. We have performed experiments, in a marble quarry, for several reasons. First, a quarry is a suitable place to produce surface waves since it presents wide areas where the surface is very flat. Second, the marble thickness underneath the surface is much larger than the penetration length of artificially created surface waves. And finally, it constitutes a system similar to the actual system we would like to represent, the earth surface. The realization of a similar experiment in a laboratory would require difficult to handle large blocks of materials, and with undesirable reflections at the borders.

The samples in our study were constructed in two stages. In a first stage, we bored 85 holes in a honeycomb lattice. When the attenuation spectra in this structure had been taken, we drilled 42 additional holes placed in the centers of the hexagons of the previous structure, so that the system became a triangular lattice with a total number of 127 holes. The holes, $160 \mathrm{~cm}$ deep and $6 \mathrm{~cm}$ in diameter, were bored with a drilling machine. The center-to-center distance between first-neighbor holes is $14 \mathrm{~cm}$. In Figs. 1(a) and 1(b) we show photographs of the quarry zone with the two types of hole distributions mentioned. The straight lines in the upper and the lower part of the structure correspond to shallow
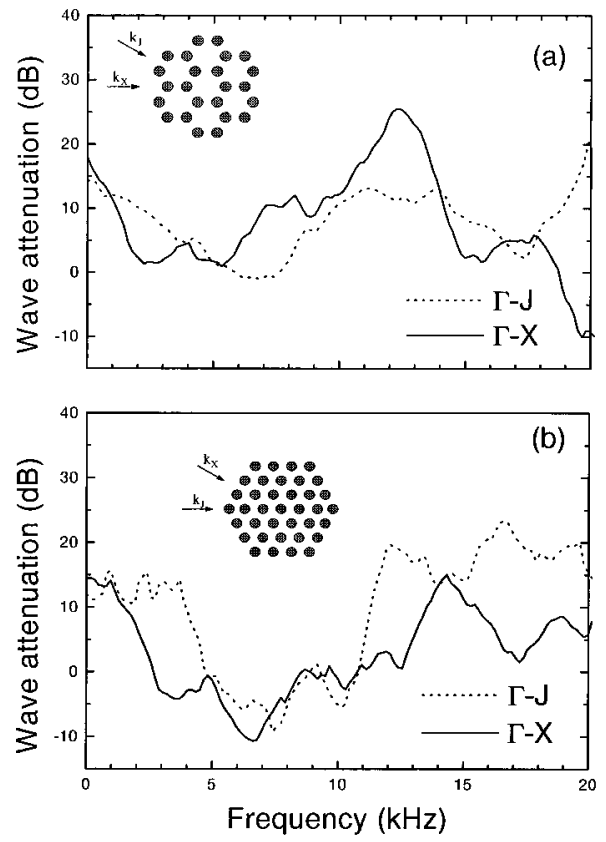

FIG. 2. (a) Attenuation spectra of the honeycomb distribution of holes for wave vector in the direction $\Gamma-X$ (full line) and $\Gamma-J$ (dotted line). (b) Same for the triangular lattice.

tracks $(1 \mathrm{~cm}$ in depth) left by the saw in the process of extraction of the marble blocks. These tracks do not influence the experiments in the range of frequencies considered in this paper.

As we progressed in drilling holes, we became aware of the existence of a sloping crack in the marble quarry at about $100 \mathrm{~cm}$ in depth at its shallowest point. The crack that has a thickness of several $\mathrm{cm}$ was filled with soil.

Surface-elastic waves are generated, each time, by the impact of a 0.5 inch steel bearing ball. This diameter size has been chosen because it produces an easily detectable signal as well as a short impact. The waves, isotropically radiated from the source, have a white spectrum containing frequencies up to $40 \mathrm{kHz}$. The wave intensity is recorded by using Brüel and Kjaer piezoelectric accelerometers vertically oriented in several configurations. These detectors work with a flat spectral response in the frequency range $0 \leqslant \nu$ $\leqslant 20 \mathrm{kHz}$. A typical seismogram produced by the ball's impact is show in Fig. 1(c). By this means, the velocity of the Rayleigh wave in plain marble can be measured: $c_{R} \approx 2960$ $\pm 30 \mathrm{~m} \mathrm{~s}^{-1}$.

One detector, placed behind the holes array, measures the signal transmitted through the structure (sample detector). The second one (reference detector), located at the same distance from the exciting impact in a clean region of the quarry, and in a direction well apart from the array of holes, records the signal that does not cross the structure. The waves are generated far from the structure in order to approximate plane waves when the wavefronts reach it. The sample detectors were located at different points to scan several directions in the irreducible part of the Brillouin Zone (BZ). In all cases we have measured the vertical component of the Rayleigh wave, i.e., the one parallel to the cylindrical holes axes.

Figures 2(a) and 2(b) show the wave attenuation (WA) spectra of the honeycomb and triangular structure. Here, 
$\mathrm{WA}=20 \times \log _{10}\left(A_{0} / A\right)$, where $A_{0}$ and $A$ are the amplitudes reaching the reference and sample detectors, respectively. We display the spectra of Rayleigh waves with $\mathbf{k}$ vectors incident along the two high-symmetry directions of the first Brillouin Zone (BZ), i.e., along $\Gamma X$ and $\Gamma J$. Let us recall that the Bravais lattice of the honeycomb structure is 1.5 times larger than the one of the triangular structure. The honeycomb structure has a smaller $\mathrm{BZ}$ and is rotated $30^{\circ}$ with respect to the triangular one. Therefore, $\Gamma X$ and $\Gamma J$ directions are interchanged in both geometries (see the insets). The negative values of WA indicate increases in signal in the sample detector due to inhomogeneities of the rock.

Regarding the honeycomb structure [see Fig. 2(a)], the spectrum along $\Gamma X$ shows two clear attenuation bands that cover a large part of spectral confidence region of the detector. The first band extends from zero up to $2 \mathrm{kHz}$, the second one covers a frequency range between 7 and $15 \mathrm{kHz}$. Along the $\Gamma J$ direction a similar spectrum is obtained; two broad bands are also defined, the first one in the range $(0-4 \mathrm{kHz})$, and the second one in the range $(8-16 \mathrm{kHz})$. A striking difference between the $\Gamma X$ and $\Gamma J$ directions, other than the widths of the peaks, is the stronger attenuation observed along $\Gamma X$. We attribute this effect to the different number of layers that the surface wave has to cross before reaching the detector (17 layers along $X$ and only 13 along $J$ ). The triangular structure [see Fig. 2(b)] shows similar features, more clearly defined. Again two broad bands appear in either direction, both being broader in the $\Gamma J$ direction and covering $0-4 \mathrm{kHz}$ and 11 to beyond $20 \mathrm{kHz}$ to be compared to $0-3$ $\mathrm{kHz}$ and $13-16 \mathrm{kHz}$ along $\Gamma X$. In comparison with the one of the honeycomb structure, the second band is shifted to higher frequencies. This band is stronger along the $\Gamma J$ direction (the direction equivalent to the $\Gamma X$ in the honeycomb) due to the different number of layers encountered by the wave. All the frequency values above mentioned have been assigned disregarding oscillations in the WA of $\pm 5 \mathrm{~dB}$ around the reference level.

The experimental observations reported above cannot be accounted for by surface roughness effects, ${ }^{14}$ and, led us to conclude that two absolute elastic-band gaps exists for the propagation of Rayleigh waves. These gaps are defined by the overlap observed between the attenuation bands along the two high-symmetry directions.

A theoretical description of the attenuation spectra implies the study of the scattering of Rayleigh waves by the array of finite air cylinders. Let $z=0$ be the stress-free surface of the semi-infinite medium occupying the half space $z \leqslant 0$. The components of the displacement fields $u_{x}$ and $u_{z}$ of a Rayleigh wave propagating along the $x$ direction with a given $k$ wave number can be expressed as

$$
u_{x}(x, z, t)=A\left[e^{-k \beta_{L} z}-\left(1-\frac{c_{R}^{2}}{2 c_{T}^{2}}\right) e^{-k \beta_{T} z}\right] \cos k\left(x-c_{R} t\right)
$$

$$
\begin{aligned}
u_{z}(x, z, t)= & A \beta_{L}\left[e^{-k \beta_{L} z}-\left(1-\frac{c_{R}^{2}}{2 c_{T}^{2}}\right)^{-1} e^{-k \beta_{T} z}\right] \\
& \times \sin k\left(x-c_{R} t\right)
\end{aligned}
$$

here $A$ is a constant, $c_{R}$ denotes the velocity of the Rayleigh wave, $c_{T}$ and $c_{L}$, the velocities of the transverse and longitudinal bulk elastic waves, respectively, while $\beta_{L}$ $=\sqrt{1-c_{R}^{2} / c_{L}^{2}}$, and $\beta_{T}=\sqrt{1-c_{R}^{2} / c_{T}^{2}}$. A calculation of the penetration depths $\left(k \beta_{L}\right)^{-1}$ and $\left(k \beta_{T}\right)^{-1}$ for the parameters of our system $\left(c_{L} \approx 6000 \mathrm{~m} \mathrm{~s}^{-1}\right.$ and $\left.c_{R} \approx 0.92 c_{T}\right)$ gives $\left(k \beta_{L}\right)^{-1} \approx 0.18 \lambda$ and $\left(k \beta_{T}\right)^{-1} \approx 0.38 \lambda .^{15}$ Therefore, the depth of the drilled cylinder establishes a critical wavelength $\lambda_{c}$ such that any wave with smaller $\lambda$ sees the cylinders as infinitely long as far as the scattering process is concerned. In our structures $\lambda_{c} \approx 4.2 \mathrm{~m}$. This implies that only those waves with frequency lower than $c_{R} / \lambda_{c} \approx 700 \mathrm{~Hz}$ experience scattering in a finite depth structure. These waves are only a minor fraction of our spectra.

The above discussion simplifies the theoretical attempt and one can consider the cylinders as semi-infinite as far as the scattering process is concerned. This calculation is beyond the scope of the present work. Instead, we have employed a simple model, based on a scalar approach, that has given us good insight into the physics involved in our experiments. The model considers a periodic three-dimensional (3D) system of cylindrical holes (scattering material $s$ ), infinite along the $z$ axis, embedded in marble (host material $h$ ). Thus, we calculate the band structure for the propagation of acoustic plane waves travelling through such a system. The corresponding equation is

$$
\nabla \cdot\left[\frac{\nabla p(\mathbf{r})}{\rho(\mathbf{r})}\right]=-\omega^{2} \frac{p(\mathbf{r})}{c^{2}(\mathbf{r}) \rho(\mathbf{r})},
$$

where $p(\mathbf{r})=\rho(\mathbf{r}) c^{2}(\mathbf{r}) \nabla \cdot \mathbf{u}(\mathbf{r}), \omega$ is the frequency of an eigenmode, and $c(\mathbf{r})$ and $\rho(\mathbf{r})$ are the wave velocity and the medium density, respectively, which are position dependent in the $2 \mathrm{D}$ space $\mathbf{r}=(x, y)$. As the wave velocity in the marble $c_{h}$ we used the one measured for the Rayleigh wave $c_{R}$. This composite material has a huge density contrast $\left(\rho_{h} / \rho_{s} \approx 2.2\right.$ $\left.\times 10^{3}\right)$ and also a large velocity contrast $\left(c_{h} / c_{s} \approx 8.8\right)$, conditions that favor the formation of full band gaps. ${ }^{6}$

We solve Eq. (3) using a variational method some of us introduced elsewhere. ${ }^{11}$ Figures 3(a) and 3(b) show the dispersion relation at filling fraction (ff) of our actual structures; $\mathrm{ff}=11.1 \%$ in the honeycomb case and $16.7 \%$ for the triangular one. The bands are extremely flat and highly degenerate. Particularly outstanding is that in both structures large gaps appear in the low-frequency region [notice the expanded scale in Figs. 3(a) and 3(b)]. This result obtained with our oversimplied model indicates that the intuitive picture of Bragg scattering fails to predict the position of attenuation bands in composite systems with huge contrasts. It also supports our claim that the attenuation bands measured in the low-frequency region does represent actual gaps of the structure for the propagation of Rayleigh waves. Attenuation bands in the low-frequency regime have also been characterized, both experimentally and theoretically, in the case of electromagnetic wave propagation through arrays of metal cylinders in air at the wavelenthgs where the dielectric constant of the metal becomes infinite. ${ }^{16}$ The calculation does not reproduce either the direction dependence of the attenuattion bands or the attenuation bands observed at high frequencies probably due to the simplifications employed. We have also calculated the behavior of the quality factor: gap/ 

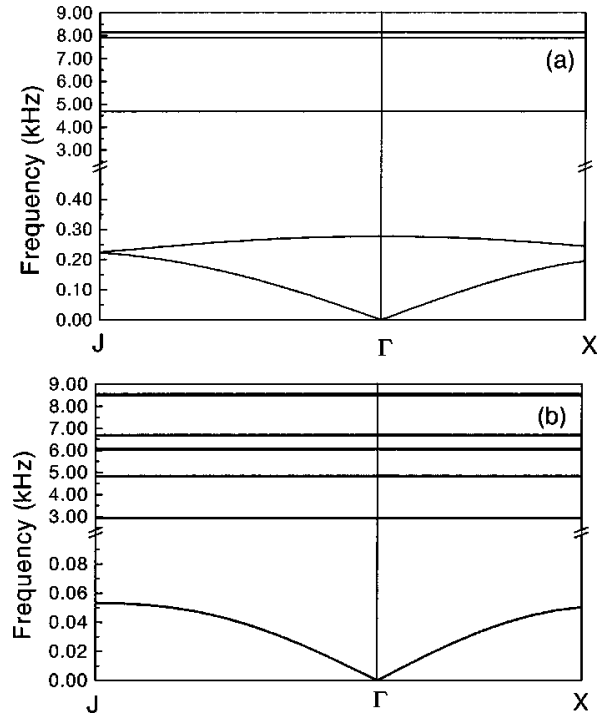

FIG. 3. (a) Band structure for scalar waves propagating in a two-dimensional elastic-band-gap crystal in a honeycomb distribution of infinite cylindrical holes in marble for a filling fraction of $11.1 \%$. (b) Same for triangular lattice with a ff of $16.7 \%$. In both calculations, we have considered $2960 \mathrm{~m} / \mathrm{s}$ as the wave velocity in marble.

midgap ratio $\left(\Delta \omega / \omega_{g}\right)$ associated with the first band gap, as a function of ff. It quickly saturates for both structures, the saturation value is $1.8(2.0)$ in the honeycomb (triangular) structure and taking place at $\mathrm{ff} \approx 1 \% \quad(\mathrm{ff} \approx 5 \%)$. This prediction, if corroborated by more realistic calculations and experiments, can have practical application since it indicates that an extremely low ff produces a band gap with large $\Delta \omega / \omega_{g}$ ratio.

In view of the results discussed above and taking into account the scaling property of this theory, it is very tempting to explore the possibility of attenuation of Rayleigh waves produced in earthquakes. Seismic movements produce surface-elastic waves of extremely low frequency, i.e., in the range $1-5 \mathrm{~s}^{-1}$. This corresponds to a $3 \pm 2 \mathrm{~s}^{-1}$ frequency range and $\Delta \omega / \omega_{g} \approx 1.3$. This frequency range can be shielded, for example, by the honeycomb symmetry using a ff around $1 \%$, that corresponds to a ratio cylinder diameter (d)-periodicity $(a) d / a=0.13$. Since the midgap value in dimensionless units at such $\mathrm{ff}$ is $\omega_{g} a / c_{R} \approx 0.20$, the optimal structure has holes $26 \mathrm{~m}$ in diameter, and $200 \mathrm{~m}$ for the first-neighbor distance. The huge contrast existing both in velocity and density makes the cylindrical holes behave as hard scatters. One can take advantage of this fact to make a proposal more feasible; one just needs to excavate annular trenches instead of digging up full cylindrical holes. Notice, however that the hole depth has to be around $1200 \mathrm{~m}$ deep in order to shield waves of $1 \mathrm{~s}^{-1}$. A word of caution is required about the above proposal, the numbers are preliminary estimates and have to be checked with improved models and further experiments.

In summary, we have presented experiments that show the attenuation of artificially generated Rayleigh waves by periodic distributions of cylindrical holes in a marble quarry. The results are explained in terms of the existence of full band gaps in these kinds of two-dimensional elastic-bandgap structures. Theoretical calculations, performed with scalar waves in their three-dimensional counterpart, also predict large gaps in the range of frequencies analyzed. Because of the scaling property associated with the equations that govern the propagation of elastic waves, the results of this paper have been employed to propose an EBG structure usable to attenuate the effects of the long-wavelength Rayleigh waves produced in seismic movements. Further studies to improve the feasibility of this application are required.

This work was partially supported by the Comisión Interministerial de Ciencia y Tecnología of Spain, Contract No. MAT97-0698-C04. We also thank the Consejo Superior de Investigaciones Científicas for the special funding to perform this experiment. The authors acknowledge J. Carrillo for the technical assistance in the drilling task, and J. P. Hernandez and F. J. García-Vidal for their critical reading of the manuscript.
*Author to whom correspondence should be addressed. Electronic address: fmese@ fis.upv.es

${ }^{\dagger}$ Author to whom correspondence should be addressed. Electronic address: jsdehesa@uamca3.fmc.uam.es

${ }^{1}$ J. D. Joannopoulos, P. R. Villeneuve, and S. Fan, Nature (London) 386, 143 (1997).

2 J. D. Joannopoulos, R. D. Meade, and R. D. Win, Photonic Crystals (Princeton University Press, Princeton, NJ, 1995).

${ }^{3}$ M. M. Sigalas and E. N. Economou, J. Sound Vib. 158, 377 (1992); E. N. Economou and M. M. Sigalas, Phys. Rev. B 48, 13434 (1993).

${ }^{4}$ M. M. Sigalas and E. N. Economou, Solid State Commun. 86, 141 (1993).

${ }^{5}$ M. S. Kushwaha, P. Halevi, L. Dobrzynski, and B. DjafariRouhani, Phys. Rev. Lett. 71, 2022 (1994); M. S. Kushwaha and P. Halevi, Appl. Phys. Lett. 64, 1085 (1994).

${ }^{6}$ M. M. Sigalas and E. N. Economou, J. Appl. Phys. 75, 2845 (1994); E. N. Economou and M. M. Sigalas, J. Acoust. Soc. Am. 95, 1734 (1994).

${ }^{7}$ M. M. Sigalas and E. N. Economou, Europhys. Lett. 36, 241
(1996); M. S. Kushwaha, Appl. Phys. Lett. 70, 3218 (1997).

${ }^{8}$ L. Ye, G. Cody, M. Zhou, P. Sheng, and A. N. Norris, Phys. Rev. Lett. 69, 3080 (1992).

${ }^{9}$ R. Martínez-Sala, J. Sancho, J. V. Sánchez, V. Gómez, J. Llinares, and F. Meseguer, Nature (London) 378, 241 (1995).

${ }^{10}$ F. R. Montero de Espinosa, E. Jiménez, and M. Torres, Phys. Rev. Lett. 80, 1208 (1998).

${ }^{11}$ J. V. Sánchez-Pérez, D. Caballero, R. Martínez-Sala, C. Rubio, J. Sánchez-Dehesa, F. Meseguer, J. Llinares, and F. Gálvez, Phys. Rev. Lett. 80, 5325 (1998).

${ }^{12}$ E. Yablonovitch, Phys. Rev. Lett. 58, 2059 (1987).

${ }^{13}$ K. Aki and P.C. Richards, Quantitative Seismology (Freeman, San Francisco, 1980).

${ }^{14}$ A. G. Eguiluz and A. Maradudin, Phys. Rev. B 28, 728 (1983).

${ }^{15}$ E. Soczkiewicz, Acustica 82, 380 (1986).

${ }^{16}$ D. R. Smith, S. Schultz, K. Kroll, M. Sigalas, K. M. Ho, and M. Soukoulis, Appl. Phys. Lett. 65, 645 (1994); D. F. Sievenpiper, E. Yablonovitch, J. N. Winn, S. Fan, P. R. Villeneuve, and J. D. Joannopoulos, Phys. Rev. Lett. 80, 2829 (1998). 